Revista Verde de Agroecologia e Desenvolvimento Sustentável

http://www.gvaa.com.br/revista/index.php/RVADS

NOTA CIENTÍFICA

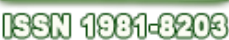

DOI: http://dx.doi.org/10.18378/rvads.v10i4.3705

\title{
Efeito do uso de diferentes compostos na produção de tomate (Lycopersicum esculentum Mill) em cultivo orgânico
}

\section{Effect of the use of different compounds in the production of tomato (Lycopersicum esculentum Mill) in organic farming}

\author{
Mauro Sergio Teodoro ${ }^{1}$; Francisco José dos Santos Seixas ${ }^{2}$; Mairla Nascimento de Lacerda ${ }^{3}$; Luma Melisa da Silva Araújo ${ }^{3}$
}

Resumo - O objetivo do presente trabalho foi avaliar, em condições de casa de vegetação, a influência de cinco compostos orgânicos na adubação de plantio e de cobertura de tomate híbrido do tipo Caqui. O delineamento experimental adotado foi o de blocos casualizados, com quatro repetições. Foram avaliados cinco tratamentos, contendo biomassa vegetal provenientes do banco de leguminosas da Embrapa Meio-Norte. As leguminosas utilizadas na confecção dos compostos foram o guandu Cultivar Fava Larga (Cajanus cajan), a Crotalária juncea (Crotalaria juncea L.), a Leucena (Leucaena leucocephala), a Gliricídia (Gliricidia sepium) e a Mucuna preta (Mucuna aterrima). A gramínea utilizada foi o capim elefante (Pennisetum purpureum). Os frutos do tomate começaram a ser colhidos no início do amadurecimento, por volta de 70 a 120 dias após o transplantio. Avaliaram-se a produção total de frutos (PTF), produção de frutos comerciais (PFC), número total de frutos (NTF), número de frutos comerciais (NFC), peso médio de frutos comerciais (PMFC) e diâmetro médio de frutos (DMF). Foram registradas ocorrências de pragas nas condições experimentais, detectando-se a presença de nematóides (Meloydogines spp) e cochonilha (Maconellicoccus hirsutus). Não houve diferença significativa entre os tratamentos estudados, entretanto, o tratamento 2 foi o que se destacou quanto as variáveis analisadas, principalmente para PTF (54,5 t/ha) e PFC (49,6 t/ha). Concluiu-se que o uso de compostos orgânicos com palhadas de leguminosas e gramíneas é uma alternativa técnica e economicamente viável, podendo reduzir o uso integral do esterco bovino na adubação de plantio e de cobertura do tomate produzido no sistema orgânico.

Palavras-chaves: resíduos orgânicos, palhadas, adubação orgânica

Abstract: - The purpose of this study was to evaluate, in greenhouse conditions, the influence of five organic compounds on fertilizing and planting tomato hybrid coverage of type Persimmons. The experimental design was randomized block, with four replicates. Five treatments were evaluated, containing vegetable biomass coming from Bank of legumes from Embrapa MeioNorte. Legumes used in the confection of the compounds were the guandu Grow Fava wide (Cajanus he), Crotalaria juncea (Crotalaria juncea 1.), the Leucena (Leucaena leucocephala), the Gliricídia (Gliricidia sepium) and Mucuna (Mucuna aterrima) black. The grass used was the elephant grass (Pennisetum purpureum). The tomato fruits began to be harvested at the beginning of the ripening, around 70 days to 120 days from transplanting. We evaluated the total production of fruits (PTF), commercial fruit production (PFC), total number of fruits (NTF), number of commercial fruits (NFC), an average weight of commercial fruits (PMFC) and average diameter of fruits (DMF). Pest occurrences were recorded in experimental conditions, detecting the presence of nematoides (Meloydogines spp) and cochineal (Maconellicoccus hirsutus). There was no significant difference between the treatments studied, however, the treatment 2 was what stood out about the variables analyzed, mostly for PTF (54.5 tha) and PFC (49.6 tha). It was concluded that the use of organic compounds with straws of legumes and grasses is a technically and economically feasible alternative, and can reduce the use of cow manure on fertilizing and planting tomato coverage produced in the organic system.

Key words: organic waste, straws, organic fertilizer

\footnotetext{
*Autor para correspondência

Recebido para publicação em 05/10/2015; aprovado em 20/11/2015

${ }_{1}^{1}$ Analista; Embrapa Meio-Norte; BR 343, Km 35, caixa postal 341, Parnaíba-PI, CEP 64200-970; mauro-sergio.teodoro@embrapa.br

${ }^{2}$ Pesquisador; Embrapa Meio-Norte; BR 343, Km 35, caixa postal 341, Parnaíba-PI, CEP 64200-970; francisco.seixas@embrapa.br

${ }^{3}$ Estudante; Universidade Estadual do Piauí - UESPI, Campus Professor Alexandre Alves de Oliveira, Av. Nossa Senhora de Fátima, S/N, Parnaíba-PI, CEP 64202-220; mairllaphb@hotmail.com, lumamelisa@hotmail.com
} 


\section{INTRODUÇÃO}

O tomateiro é considerado, dentre as hortaliças, uma das espécies mais exigentes em adubação. Conhecer as exigências nutricionais, os principais sintomas de deficiências e o modo de corrigi-las é fundamental para o êxito da cultura (SILVA; GIORDANO, 2000).

No sistema convencional, são utilizados adubos químicos de alta solubilidade, que são agentes degradantes da matéria orgânica (LUZ et al., 2007), além de contaminantes do solo, lençol freático e dos rios (ROCHA et al., 2014). Na produção orgânica são utilizados adubos de baixa solubilidade e com altos teores de matéria orgânica, que visam estruturar a microbiologia do solo (LUZ et al., 2007).

A agricultura e a pecuária produzem quantidades de resíduos, como dejetos de animais e restos de culturas, palhas e resíduos agroindustriais, os quais, em alguns casos, provocam sérios prejuízos e problemas de poluição. Muitos desses resíduos são perdidos por não serem coletados e reciclados ou por serem destruídos pelas queimadas. Todavia, quando manipulados adequadamente, podem suprir aos sistemas agrícolas, boa parte da demanda de insumos sem afetar os recursos do solo e do ambiente (TEIXEIRA, 2002).

Para o tomateiro, hortícola de alto valor econômico, a adubação orgânica tem sido normalmente praticada como fonte de nutrientes, dentre eles o nitrogênio, visando suprir parte das necessidades nutricionais dessa espécie (MELLO; VITTI, 2002). Neste contexto, o composto orgânico é extremamente importante, pois constitui a base da adubação orgânica.

A prática da compostagem orgânica, em linhas gerais, consiste na mistura de estercos animais com resíduos de origem vegetal (KIEHL, 2002). Porém, apesar das vantagens de sua utilização, a aquisição de esterco de fontes externas para confecção do composto orgânico, pode tornarse inviável economicamente, constituindo-se em prática insustentável.

Guelber Sales et al. (2008) salienta que a compostagem é importante nos sistemas agrícolas de produção, devido a sua capacidade de integrar atividades, além de otimizar a ciclagem da matéria orgânica disponível. Segundo os autores, a diversificação de inoculantes para a montagem das "leiras" pode ser uma alternativa promissora para incrementar a ciclagem de nutrientes, ampliando a sustentabilidade do processo de compostagem na propriedade rural.

Entretanto, por não ser um produto único, sua qualidade pode variar de acordo com os resíduos orgânicos e os processos empregados (VALENTE et al., 2009), justificando-se estudos adicionais que visem diversificar a base da compostagem por meio da seleção de resíduos disponíveis e de fácil produção na unidade rural (GUELBER SALES et al., 2008).
De acordo com Leal et al. (2007), uma das alternativas para reduzir o uso de esterco no processo de compostagem é a utilização de leguminosas como fonte de biomassa vegetal para a confecção de composto orgânico. O mesmo autor descreve um composto orgânico eficiente para a produção de hortaliças, obtido por meio da mistura de palhada de capim napier (Pennisetum purpureum) e da leguminosa Crotalária juncea, sem adição de outros inoculantes.

Dentre as leguminosas que apresentam bom potencial de produção de biomassa e que podem ser utilizadas na confecção de compostos orgânicos destacam-se a Gliricídia (BARRETO; FERNANDES, 2001), Crotalária juncea (LEAL et al., 2009), Mucuna preta (FORMENTINI et al., 2008), Guandu anão Cv Fava Larga (CESAR et al., 2011), e a Leucena (ALENCAR; GUSS, 1991).

O objetivo deste trabalho foi avaliar a influência de cinco compostos orgânicos, produzidos com diferentes biomassas vegetais na adubação do tomate híbrido tipo Caqui "Dominador F1".

\section{MATERIAL E MÉTODOS}

O trabalho foi desenvolvido durante o período de maio a setembro de 2013, na Embrapa Meio-Norte/UEP

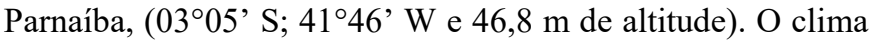
da região é do tipo $\mathrm{C}_{1} \mathrm{dA}$ 'a' (THORNTHWAITE e MATHER, 1955), caracterizado como subúmido seco, megatérmico, com pequeno excedente hídrico e uma concentração de $29,7 \%$ da evapotranspiração potencial no trimestre outubro, novembro e dezembro, com umidade média relativa do ar de 77,5\%, precipitação média de 1107 $\mathrm{mm}$, e temperatura média de $27,6^{\circ} \mathrm{C}$ (BASTOS et al., 2012).

A casa de vegetação utilizada para a implantação do ensaio de tomate orgânico foi construída com estrutura de madeira, paredes laterais de tela antiafídica e teto com telado do tipo sombrite $(50 \%)$. A cultivar utilizada foi o tomate híbrido tipo Caqui "Dominador F1" da Topseed, com ciclo médio de 120 dias. Todas as leguminosas utilizadas para a confecção dos compostos orgânicos nesse ensaio foram cultivadas na "Vitrine permanente de tecnologias para a transição agroecológica - adubos verdes para o manejo da matéria orgânica e fertilidade do solo", instalada na Embrapa Meio-Norte/UEP de Parnaíba. Essa vitrine foi instalada com o objetivo de avaliar a adaptação de leguminosas anuais e perenes, e também, de algumas gramíneas, frente às condições edafoclimáticas da região Meio-Norte para sua difusão junto aos agricultores.

O delineamento experimental adotado foi o de blocos casualizados, com cinco tratamentos (compostos orgânicos) e quatro repetições. Os compostos orgânicos constituíram-se de $35 \%$ de gramínea - Penisetum purpureum - (PP) associada a $30 \%$ de esterco bovino (EB) e $35 \%$ de uma leguminosa (Tabela 1).

Tabela 1 - Diferentes tratamentos/compostos orgânicos utilizados na produção de tomate. Parnaíba/PI. 2013

\begin{tabular}{cl}
\hline Tratamento & Composição \\
\hline T1 & $35 \%$ Guandu Cultivar Fava Larga $+35 \% \mathrm{PP}+30 \% \mathrm{~EB}$ \\
T2 & $35 \%$ Crotaláriajuncea $+35 \% \mathrm{PP}+30 \% \mathrm{~EB}$ \\
$\mathrm{~T} 3$ & $35 \%$ Leucena $+35 \% \mathrm{PP}+30 \% \mathrm{~EB}$ \\
$\mathrm{~T} 4$ & $35 \%$ Gliricídia $+35 \% \mathrm{PP}+30 \% \mathrm{~EB}$ \\
$\mathrm{~T} 5$ & $35 \%$ Mucuna preta $+35 \% \mathrm{PP}+30 \% \mathrm{~EB}$ \\
\hline
\end{tabular}


As mudas de tomate foram produzidas na Embrapa Meio-Norte/UEP Parnaíba, utilizando-se como substrato o composto orgânico produzido localmente com palhadas vegetais de gramíneas e leguminosas, além do esterco bovino. A proporção utilizada na confecção do composto foi de $1 / 3$ para cada material. Após maturado, este substrato foi peneirado (malha 3,70 mm). Após preenchimento dos copos descartáveis $(200 \mathrm{ml})$ utilizou-se uma semente por recipiente. As mudas foram conduzidas em viveiro e transplantadas para a casa de vegetação 20 dias após a semeadura, quando já apresentavam três a quatro folhas definitivas.

Os canteiros foram preparados manualmente, tendo por espaçamento $1,00 \mathrm{~m}$ entre linhas e $0,40 \mathrm{~m}$ entre plantas, totalizando 10 plantas por parcela, inseridas em covas de 20 $\mathrm{cm}$ de profundidade. Na adubação de plantio foram utilizados 2 litros do composto orgânico. As análises das propriedades químicas dos diferentes tipos de compostos orgânicos encontram-se na Tabela 2.

Tabela 2 - Composição química dos compostos orgânicos utilizados. Parnaíba/PI. 2013

\begin{tabular}{|c|c|c|c|c|c|}
\hline COMPONENTE & T1 & T2 & T3 & T4 & T5 \\
\hline pH (água 1:10) & 8,0 & 8,3 & 8,7 & 8,8 & 8,1 \\
\hline Cálcio $(\mathrm{g} / \mathrm{kg})^{(1)}$ & 9,7 & 9,6 & 13,3 & 13,7 & 10,9 \\
\hline Fósforo (g/kg) & 5,3 & 5,6 & 6,0 & 8,1 & 6,5 \\
\hline Relação cálcio/magnésio & 2,55 & 2,53 & 3,02 & 3,11 & 2,59 \\
\hline Magnésio (g de $\mathrm{Mg} / \mathrm{kg})^{(1)}$ & 3,8 & 3,8 & 4,4 & 4,4 & 4,2 \\
\hline Sódio (mg de $\mathrm{Na} / \mathrm{kg})^{(1)}$ & 755 & 805 & $<1,0^{(2)}$ & 908 & $<10,0^{(2)}$ \\
\hline Potássio (mg de $\mathrm{K} / \mathrm{kg})^{(1)}$ & 5452 & 5447 & 5199 & 4950 & 5197 \\
\hline Zinco (mg de Zn/kg) ${ }^{(1)}$ & 134 & 140 & 130 & 159 & 148 \\
\hline Ferro $(\mathrm{mg}$ de $\mathrm{Fe} / \mathrm{kg}$ ) & 769 & 580 & 916 & 904 & 1264 \\
\hline Manganês (mg de $\mathrm{Mn} / \mathrm{kg})^{(1)}$ & 253 & 258 & 255 & 378 & 296 \\
\hline Cobre $(\mathrm{mg} \mathrm{de} \mathrm{Cu} / \mathrm{kg})^{(1)}$ & 28,4 & 26,6 & 27,7 & 38,6 & 32,6 \\
\hline Carbono orgânico $(\mathrm{g} \text { de } \mathrm{C} / \mathrm{kg})^{(1)}$ & 321 & 206 & 263 & 242 & 218 \\
\hline Nitrogênio Kjeldahl (g de N/kg) ${ }^{(1)}$ & 24,9 & 19,6 & 21,5 & 21,5 & 20,5 \\
\hline Relação C/N & 12,89 & 10,51 & 12,23 & 11,26 & 10,63 \\
\hline
\end{tabular}

${ }^{(1)}$ Resultados expressos em base seca.

(2) Não quantificado, menor do que o limite de quantificação.

O sistema de irrigação utilizado foi o de gotejamento com fornecimento de água de 4L/hora/planta. Todos os canteiros receberam cobertura morta com palhas de carnaúba (Copernicia prunifera), com o intuito de manter a umidade dos canteiros e diminuir a temperatura do solo.

As plantas do tomate foram tutoradas individualmente com fitas plásticas, em haste única, eliminando-se todas as brotações laterais das axilas das folhas. O amarrio acompanhou o tutoramento, sendo realizados semanalmente e de acordo com o desenvolvimento do tomateiro. Após a emissão do $7^{\circ}$ cacho foi realizada a poda da haste principal.

Foram realizadas adubações de cobertura aos 30, 50 e 70 dias, com os mesmos compostos orgânicos utilizados nas adubações de fundação junto ao "colo", porém, em menor quantidade (1 litro).

Foi realizado o controle de lagartas com o uso de produto a base de Bacilus thuringiensis permitido para a agricultura orgânica.

As avaliações agronômicas foram realizadas com base de 6 plantas por parcela $\left(2,4 \mathrm{~m}^{2}\right.$ de área útil/parcela), descartando-se as plantas localizadas nas bordaduras. Os dados foram anotados individualmente por planta e as médias foram calculadas por parcela. Avaliaram-se a produção total de frutos (PTF); produção de frutos comerciais (PFC); número total de frutos (NTF); número de frutos comerciais (NFC); peso médio de frutos comerciais (PMFC), e diâmetro médio de frutos (DMF).

Os dados obtidos foram submetidos à análise de variância, utilizando-se o software "ASSISTAT" para medir a significância da variação não ocasional dos valores e o teste de Tukey, ao nível de 5\% de probabilidade, para o contraste de médias.

\section{RESULTADOS E DISCUSSÃO}

Os frutos de tomate começaram a ser colhidos no início do processo de amadurecimento, quando apresentaram coloração rosada e o tempo gasto do transplantio ao início da colheita variou de 70 a 120 dias. Segundo Souza (2010), essa variação depende da variedade, da região e da época de plantio.

Na Tabela 3 são apresentados os resultados dos componentes avaliados no período do experimento. Observou-se que não houve diferença significativa entre os tratamentos em relação às variáveis analisadas. 
Tabela 3 - Média da Produção total de frutos (PTF), produção de frutos comerciais (PFC), número total de frutos (NTF), número de frutos comerciais (NFC), peso médio de frutos comerciais (PMFC) e diâmetro médio de frutos de tomate sob efeito de diferentes compostos orgânicos. Parnaíba/PI. 2013

\begin{tabular}{|c|c|c|c|c|c|c|}
\hline Tratamentos & $\begin{array}{c}\text { PTF } \\
\text { (t/ha) }\end{array}$ & $\begin{array}{l}\text { PFC } \\
\text { (t/ha) }\end{array}$ & $\begin{array}{c}\text { NTF } \\
\text { (frutos/m2) }\end{array}$ & $\begin{array}{c}\text { NFC } \\
\text { (frutos/m2) }\end{array}$ & $\begin{array}{c}\text { PMFC } \\
\text { (g/fruto) }\end{array}$ & $\begin{array}{r}\text { DMF } \\
(\mathbf{c m})\end{array}$ \\
\hline 1 & $34,8 \mathrm{a}$ & $31,8 \mathrm{a}$ & $37,71 \mathrm{a}$ & $34,16 \mathrm{a}$ & $87,43 \mathrm{a}$ & $5,20 \mathrm{a}$ \\
\hline 2 & $54,5 \mathrm{a}$ & $49,6 \mathrm{a}$ & $58,54 \mathrm{a}$ & $52,92 \mathrm{a}$ & $94,68 \mathrm{a}$ & $5,28 \mathrm{a}$ \\
\hline 3 & $28,6 \mathrm{a}$ & $27,6 \mathrm{a}$ & $33,54 \mathrm{a}$ & $32,29 \mathrm{a}$ & $80,70 \mathrm{a}$ & $5,06 \mathrm{a}$ \\
\hline 4 & $49,2 \mathrm{a}$ & $45,9 \mathrm{a}$ & $55,10 \mathrm{a}$ & $51,56 \mathrm{a}$ & 89,36 a & $5,19 \mathrm{a}$ \\
\hline 5 & $38,1 \mathrm{a}$ & $34,7 \mathrm{a}$ & $40,62 \mathrm{a}$ & $37,71 \mathrm{a}$ & $91,71 \mathrm{a}$ & $5,26 \mathrm{a}$ \\
\hline Média & $41,1 \mathrm{a}$ & $37,9 \mathrm{a}$ & $45,10 \mathrm{a}$ & $41,73 \mathrm{a}$ & $88,78 \mathrm{a}$ & $5,20 \mathrm{a}$ \\
\hline $\mathrm{CV}$ & 43,34 & 45,11 & 40,86 & 42,44 & 14,03 & 5,40 \\
\hline
\end{tabular}

Nas colunas, médias seguidas de letras iguais não diferem $(\mathrm{P}>0,05)$ pelo teste de Tukey.

O tratamento 2 apresentou valores maiores para PTF $(54,5 \mathrm{t} / \mathrm{ha})$ e PFC (49,6 t/ha), mas não diferiram dos demais tratamentos (Tabela 3). Independentemente dos tratamentos aplicados, os resultados obtidos em cultivo convencional do tomateiro são superiores aos deste trabalho, registrando-se média de 115,3 t/ha em ambiente protegido com o híbrido EF-50 por Fayadet al. (2001), e média de 85,4 t/ha segundo Carrijo et al. (2004). Entretanto, considerando-se as características da espécie utilizada neste ensaio, esses níveis de rendimento podem ser considerados satisfatórios, dentro dos princípios da produção orgânica de alimentos.

O rendimento da cultura do tomate em plantios a campo de sistemas orgânicos tem variado de 30 a 40 t/ha (SOUZA, 2010). Em estudo realizado pelo Instituto Capixaba de Pesquisa e Extensão Rural (INCAPER), Souza, (2002) obteve uma produtividade média de 34,5 t/ha de frutos comerciais de tomateiro orgânico, também em condições de campo, ao longo de oito anos. O mesmo autor afirma que em plantios orgânicos, realizados em ambiente protegido, a produtividade comercial de frutos, tem variado de 50 a 60 t/ha, em propriedades de agricultores orgânicos.

Carvalho e Tessarioli Neto (2005) e Marim et al. (2005), observaram maior produção de frutos de melhor classificação com a condução de uma haste por planta. Estes métodos de condução com uma ou duas hastes por planta, bem como as diferentes cultivares e tipos de tomate existentes podem ter respostas diferentes quanto aos métodos de tutoramento.

Nesse estudo, com uma planta por cova conduzida em haste única, as maiores médias de NTF e de NFC por planta foram obtidos nos tratamentos 2 e 4 , com valores de 58,5 e 55,1 frutos por $\mathrm{m}^{2}$, e de 52,9 e de 51,7 frutos por $\mathrm{m}^{2}$, respectivamente. No entanto, não diferiram estatisticamente dos demais tratamentos. Gravina et al. (2010), obtiveram número total de frutos por unidade de área com média de 61,4 frutos por $\mathrm{m}^{2}$, resultado semelhante ao obtido por Bogiani et al. (2008) ao analisarem diferentes sistema de cultivo e coberturas de solo.

Charlo et al. (2009) salientam que a condução de duas plantas por cova leva a menor número de frutos por planta, ocorrendo 39,17 frutos por planta quando orientada com 1 haste e 46,96 frutos por planta quando orientada com duas hastes, independentemente do tamanho dos frutos.
Carvalho e Tessarioli Neto (2005) também verificaram menor número de frutos por planta quando conduzidas com uma haste, em comparação às conduzidas com duas hastes. Segundo os autores, isso se deve ao fato de que plantas conduzidas com duas hastes emitem maior número de rácemos e, consequentemente, produzem maior número de frutos.

Observa-se a mesma tendência também para a variável PMFC, obtendo-se valores de 94,68 g/fruto e de $89,36 \mathrm{~g} /$ fruto, respectivamente para os tratamentos 2 e 4 . Salienta-se que o tratamento 5 apresentou resultado semelhante ao tratamento 4, porém, não existindo diferença significativa $(\mathrm{P}>0,05)$ entre ambos.

Foi observado que não houve diferença significativa $(\mathrm{P}>0,05)$ no diâmetro dos frutos (DMF), oriundos dos tratamentos, classificados quanto ao tamanho na classe 50, segundo a CEAGESP (2003). A maior média foi obtida no tratamento 2, com valor encontrado de $5,28 \mathrm{~cm}$ e a menor média foi a obtida no tratamento 3 , com $5,06 \mathrm{~cm}$. Gomes et al. (2012) obtiveram diâmetros médios de $5,89 \mathrm{~cm}$ em tomate cultivado em sistema de monocultivo e de $5,43 \mathrm{~cm}$ em sistema de policultivo.

Foram registradas as ocorrências de pragas, tais como nematóide (Meloidogyne spp), cochonilha (Maconellicoccus hirsutus) e lagartas. O ataque de pragas, ainda que em ambiente protegido, constitui um grande desafio a ser vencido na produção de tomate orgânico, e em nosso estudo, essa ocorrência pode ter influenciado nos resultados obtidos.

\section{CONCLUSÕES}

O uso de compostos orgânicos confeccionados com palhada de leguminosas e palhada de capim Napier (Pennisetum purpureum) pode ser uma alternativa viável ao uso do esterco bovino na adubação orgânica.

\section{REFERÊNCIAS BIBLIOGRÁFICAS}

ALENCAR, J.A. de; GUSS, A. Efeito da frequência de corte sobre a produção de matéria seca e proteína bruta da cunhã (Clitoria ternatea) e da leucena (Leucaena leucocephala). Vitória: EMCAPA, 1991, 5p. (EMCAPA. Comunicado Técnico, 65). 
BARRETO, A. C.; FERNANDES, M. F. Cultivo de Gliricidia sepium e Leucaena leucocephala em alamedas visando à melhoria dos solos dos tabuleiros costeiros. Pesquisa Agropecuária Brasileira, 36 (10):1287-1293. 2001

BASTOS, E. A.; ANDRADE JUNIOR, A. S. de; RODRIGUES, B. H. N. Boletim agrometeorológico de 2011 para o município de Parnaíba, Piauí. Teresina: Embrapa Meio-Norte, 2012. 37p. (Embrapa Meio-Norte. Documentos, 221).

BOGIANI, J. C.; ANTON, C. S.; SELEGUINI, A.; FARIA JÚNIOR, M. J. A.; SENO, S. Poda apical, densidade de plantas e cobertura plástica do solo na produtividade do tomateiro em cultivo protegido. Bragantina, Campinas, v.67, n.1, p.145-151. 2008.

CARRIJO, O. A.; VIDAL, M. C.; REIS, N. V. B.; SOUZA, R. B.; MAKISHIMA, N. Produtividade do tomateiro em diferentes substratos e modelos de casas de vegetação. Horticultura Brasileira, v.22, n.1, 2004, p.05-09.

CARVALHO, L. A; TESSARIOLI, NETO. J. Produtividade de tomate em ambiente protegido, em função do espaçamento e número de ramos por planta. Horticultura Brasileira 23: 986-989. 2005.

CEAGESP - Companhia de entrepostos e armazéns gerais de São Paulo. Programa brasileiro para a modernização da horticultura: Normas para a classificação do tomate. Centro de Qualidade em Horticultura, São Paulo, 26p. 2003.

CESAR, M. N. Z.; GUERRA, J. G. M.; RIBEIRO, R. de L. D.; URQUIAGA, S. S. C.; PADOVAN, M. P. Performance de adubos verdes cultivados em duas épocas do ano no Cerrado do Mato Grosso do Sul. Rev. Bras. de Agroecologia. 6(2): 159-169 (2011)

CHARLO, H. C. O; SOUZA, S. C; CASTOLDI, R.; BRAZ, L. T. Desempenho e qualidade de frutos de tomateiro em cultivo protegido com diferentes números de hastes. Horticultura Brasileira 27: 144-149. 2009.

FAYAD, J. A.; FONTES, P. C. R.; CARDOSO, A. A.; FINGER, F. L.; FERREIRA, F. A. Crescimento e produção do tomateiro cultivado sob condições de campo e de ambiente protegido. Horticultura Brasileira, v.19, n.3, 2001, p.365-370.

FORMENTINI, E. A.; LÓSS, F. R.; BAYERL, M. P.; LOVATI, R. D.; BAPTISTA, E. Cartilha sobre adubação verde e compostagem. Vitória, 2008, 27p.

GOMES, F. B; FORTUNATO, L. J; PACHECO, A. L. V; AZEVEDO, L. H; FREITAS, N.; HOMMA, S. K. Incidência de pragas e desempenho produtivo de tomateiro orgânico em monocultivo e policultivo. Horticultura Brasileira 30: 756761. 2012.

GRAVINA, C. S; MAROUELLI, W. A; JUNQUEIRA, A. M. R; SOUZA, R. F; ARAÚJO, T. A. Produção de tomate orgânico sob diferentes manejos de irrigação. Horticultura Brasileira 28: S2834-S2842. 2010.

GUELBER SALES, M. N. et al. Unidade experimental de produção animal agroecológica: uma abordagem sistêmica na construção do conhecimento. In: PADOVAN, M. P. et al Pesquisa Agroecológica Capixaba. Vitória, ES: INCAPER,
2008. p. $48-58$

KIEHL, E. J. Manual de Compostagem: Maturação e Qualidade do Composto. Piracicaba: $3^{\mathrm{a}}$ Edição do Autor, 2002. 171p.

LEAL, M. A. A et al. Utilização de compostos orgânicos como substratos na produção de mudas de hortaliças. Horticultura Brasileira, Brasília. v. 25, n. 3, p. 392-395. 2007.

LEAL, M. A. A. et al. Adubação orgânica de beterraba com composto obtido a partir da mistura de palhada de gramínea e de leguminosa. Seropédica, RJ: Embrapa Agrobiologia, 2009. (Boletim da Embrapa, 43).

LUZ, J. M. Q.; SHINZATO, A. V.; SILVA, M. A. D. Comparação dos sistemas de produção de tomate convencional e orgânico em cultivo protegido. Bioscience journal. Uberlândia, v. 23, n. 2, p. 7-15, abr./jun. 2007.

MARIM, B. G.; SILVA, D. J. H.; GUIMARÃES, M. A.; BELFORT, G. Sistemas de tutoramento e condução do tomateiro visando produção de frutos para consumo in natura. Horticultura Brasileira 23: 951-955. 2005.

MELLO, S. C.; VITTI, G. C. Influência de materiais orgânicos no desenvolvimento do tomateiro e nas características químicas do solo em ambiente protegido. Horticultura Brasileira, Brasília, v. 20, n 3, p. 452-458, 2002.

ROCHA, M. de S.; PEREIRA, E. S.; TEIXEIRA, V. M. Avaliação de impactos ambientais na agricultura familiar de Colorado do Oeste, Rondônia. V Congresso Brasileiro de Gestão Ambiental. Belo Horizonte/MG - 24 a 27/11/2014. In V Congresso Brasileiro de Gestão Ambiental, Anais...Belo Horizonte/MG, 2014.

SILVA, J. B. C.; GIORDANO, L. B. Produção mundial e nacional. In: SILVA, J. B. C.; GIORDANO, L. B. Org. Tomate para processamento industrial. Brasília: Embrapa Comunicação para Transferência de Tecnologia/Embrapa Hortaliças, 2000. P.8-11.

SOUZA, J. L. de. Curso Técnico de Agricultura Orgânica. Domingos Martins: INCAPER. 2002, 262 p. (Apostila mimeografado).

SOUZA, J. L. de. Sistema orgânico de produção de tomate. In: INSTITUTO CAPIXABA DE PESQUISA, ASSISTÊNCIA TÉCNICA E EXTENSÃO RURAL. Tomate.Vitória, ES: Incaper, p. 35 - 67. 2010. ISBN 978-8589724-17-3.

TEIXEIRA, R. F. F. Compostagem. In: HAMMES, V.S. (Org.) Educação ambiental para o desenvolvimento sustentável. Brasília: Embrapa Informação Tecnológica, v.5, p.120-123. 2002.

THORNTHWAITE, C. W.; MATHER, J. R. The water balance. Centerton: Drexel Institute of technology, 104 p. (Drexel Institute of technology. Publication in climatology, v. 8, n. 1). 1955.

VALENTE, B. S.; XAVIER, E. G.; MORSELLI, T. B. G. A.; JAHNKE, D. S.; BRUM JUNIOR, B. de S.; CABRERA, B. R.; MORAES, P. de O.; LOPES, D.C.N. Fatores que afetam o desenvolvimento da compostagem de resíduos orgânicos. Arch. Zootec. 58 (R): 59-85. 2009. 